\title{
Clinical implications and molecular mechanisms of immunoparalysis after cardiopulmonary bypass
}

\author{
Timothy T. Cornell, MD, ${ }^{a}$ Lei Sun, PhD, ${ }^{a}$ Mark W. Hall, MD, ${ }^{b}$ James G. Gurney, PhD, ${ }^{c}$ \\ Matthew J. Ashbrook, BS, ${ }^{a}$ Richard G. Ohye, MD, ${ }^{c}$ and Thomas P. Shanley, MD ${ }^{\mathrm{a}}$
}

\begin{abstract}
Objective: We used a whole blood assay to characterize the immune system's response after cardiopulmonary bypass $(\mathrm{CPB})$ in children to identify the risk for postoperative infections. We assessed the impact of CPB on histone methylation as a potential mechanism for altering gene expression necessary for the immune system's capacity to defend against infections.
\end{abstract}

\begin{abstract}
Methods: We prospectively enrolled patients less than 18 years old undergoing heart surgery requiring CPB at C.S. Mott Children's Hospital. Blood was obtained from patients before CPB, on CPB, and on postoperative days 1, 3, and 5. Ex vivo lipopolysaccharide-induced tumor necrosis factor-alpha production measured the capacity of the immune system. Serum cytokines were measured using a multiplex assay. Chromatin immunoprecipitation to detect histone modifications at the interleukin (IL) 10 promoter was performed on circulating mononuclear cells from a subgroup of patients.
\end{abstract}

Results: We enrolled 92 patients, and postoperative day 1 samples identified a subpopulation of immunocompetent patients at low risk for infections with a specificity of 93\% (confidence interval [CI], 83\%-98\%) and a negative predictive value of $88 \%(\mathrm{CI}, 77 \%-95 \% ; P=.006)$. Patients classified as immunoparalyzed had serum IL-10 levels 2.4-fold higher than the immunocompetent group (mean, $14.3 \pm 18.3 \mathrm{pg} / \mathrm{mL}$ vs $6.0 \pm 5.0$ $\mathrm{pg} / \mathrm{mL} ; P=.01$ ). In a subgroup of patients, we identified a greater percent of the "gene on" epigenetic signature, H3K4me3, associated with the IL-10 promoter after CPB.

Conclusions: Our data demonstrate that immunophenotyping patients after CPB can predict their risk for the development of postoperative infections. Novel mechanistic data suggest that CPB affects epigenetic alterations in IL-10 gene regulation. (J Thorac Cardiovasc Surg 2012;143:1160-6)

Supplemental material is available online.

Morbidity and mortality associated with children requiring cardiopulmonary bypass (CPB) is in part attributed to postoperative infections. Risk factors for these infections are younger age, longer operative and $\mathrm{CPB}$ times, complex

From the Division of Pediatric Critical Care Medicine, ${ }^{a}$ Department of Pediatrics and Communicable Diseases, C.S. Mott Children's Hospital University of Michigan Medical School, Ann Arbor, Mich; Section of Critical Care Medicine, ${ }^{\mathrm{b}}$ Nationwide Children's Hospital Department of Pediatrics, The Ohio State University College of Medicine, The Research Institute at Nationwide Children's Hospital, Columbus, Ohio; and the Michigan Congenital Heart Outcomes Research and Discovery Program (M-CHORD), ${ }^{\mathrm{c}}$ Division of Cardiology and Section of Cardiac Surgery, C.S. Mott Children's Hospital, University of Michigan Medical School, Ann Arbor, Mich.

This project was supported by National Institutes of Health grants K12GM076344 (to T.T.C.), K08HD062142 (to T.T.C.), UL1RR024986 (to T.P.S.), and 1R01HL097361-01 (to T.P.S.).

Disclosures: Authors have nothing to disclose with regard to commercial support.

Received for publication Jan 24, 2011; revisions received July 14, 2011; accepted for publication Sept 15, 2011; available ahead of print Oct 13, 2011

Address for reprints: Timothy T. Cornell, MD, Division of Critical Care Medicine, C.S. Mott Children's Hospital, F-6882, 1500 East Medical Center Dr, Ann Arbor, MI 48109-0243 (E-mail: ttcornel@med.umich.edu).

$0022-5223 / \$ 36.00$

Copyright $($ c 2012 by The American Association for Thoracic Surgery doi:10.1016/j.jtcvs.2011.09.011 surgical repair, and extended duration of mechanical ventilation. ${ }^{1-3}$ Circulating leukocytes, including peripheral blood monocytes, are activated by the CPB circuit ${ }^{4}$ resulting in a proinflammatory response that is responsible for the early physiologic derangements observed after CPB. A compensatory anti-inflammatory response aimed at countering proinflammation is necessary to reestablish homeostasis. An exaggerated or prolonged anti-inflammatory response can impair immune defense, rendering the host susceptible to infections.

Increased investigation of the immunobiology of the prolonged anti-inflammatory response has uncovered the presence of "deactivated" peripheral blood monocytes that have an attenuated response to ex vivo lipopolysaccharide (LPS) challenge with tumor necrosis factor-alpha (TNF- $\alpha$ ) and a decrease in the cell surface major histocompatibility complex II molecule, human leukocyte antigen (HLA) DR. ${ }^{5}$ The term "immunoparalysis" refers to the combination of decreased ex vivo LPS responsiveness and HLA-DR expression. ${ }^{6}$ The decrease in HLA-DR expression on isolated monocytes resulting in an immunoparalyzed state after CPB has been observed in children ${ }^{7}$ and is associated with increased serum interleukin (IL) 10 levels. ${ }^{8}$ However, HLA-DR receptor expression denotes a phenotypic characteristic but does not reflect the functional capacity of the innate immune system. 

Abbreviations and Acronyms
ChIP = chromatin immunoprecipitation
$\mathrm{CI}=$ confidence interval
$\mathrm{CPB}=$ cardiopulmonary bypass
HLA = human leukocyte antigen
IL $\quad=$ interleukin
LPS $=$ lipopolysaccharide
RACHS-1 = Risk Adjustment in Congenital Heart Surgery
TNF- $\alpha=$ tumor necrosis factor-alpha

In separate work examining patients with sepsis, the inflammatory response of sepsis is associated with significant repression of many genes expressed by both innate and adaptive immunity and increased expression of IL-10. ${ }^{9}$ Experimental data suggest such an expression pattern is mediated via epigenetically regulated histone methylation and ultimately leads to an immunoparalyzed state. ${ }^{10}$

The aim of our study was to investigate the impact of immunoparalysis after $\mathrm{CPB}$ in pediatric patients using a functional ex vivo whole blood assay to immunophenotype, or stratify, patients. We further investigated the impact of CPB on histone methylation at the IL-10 promoter as a potential mechanistic contributor to immunoparalysis.

\section{METHODS}

\section{Patient Selection}

This prospective cohort study was conducted at C.S. Mott Children's Hospital between September 2005 and March 2009 and was approved by the Institutional Review Board. Patient information was de-identified consistent with Health Insurance Portability and Accountability Act regulations. Informed consent was obtained from parents of patients less than 18 years of age before surgery. Patients were excluded if they had a diagnosis consistent with immune suppression, an active infection as determined by positive cultures before surgery, or no vascular access for blood sampling.

\section{Anesthesia}

Anesthesia was induced with sevoflurane. Neuromuscular blockade was achieved with pancruonium. Anesthesia was maintained throughout the case with fentanyl and isoflurane. All patients received intraoperative antibiotics (cefazolin).

\section{Cardiopulmonary Bypass}

The CPB circuit was primed with Normosol solution (Hospira, Inc, Lake Forest, Ill), colloid, sodium barcarbonate, mannitol $(0.5 \mathrm{~g} / \mathrm{kg})$, and heparin. For neonates and infants, packed red blood cells were added to the circuit prime using white blood cell filters. Gas exchange was achieved with a hollow-fiber membrane oxygenator in circuit with nonpulsatile occlusive roller pumps. Pump flow was adjusted to keep systemic perfusion pressures between 20 and $40 \mathrm{~mm} \mathrm{Hg}$ for neonates and infants and between 30 and $50 \mathrm{~mm} \mathrm{Hg}$ for older children. Full flow on CPB was between 2.4 and $3.0 \mathrm{~L} \cdot \mathrm{min}^{-1} \cdot \mathrm{m}^{-2}$. The patient's core temperature was controlled by a heat exchanger in the CPB circuit and was monitored with esophageal, skin, and rectal or bladder temperature probes. All patients received heparin (400 IU/kg) before aortic cannulation.

\section{Clinical Data}

Demographic data, duration of $\mathrm{CPB}$, and intraoperative medications were collected after arrival in the intensive care unit. Laboratory values, medications, blood products, organ dysfunction scores, and culture results were collected every morning for the duration of the intensive care unit stay. Surgical complexity was determined by the Risk Adjustment in Congenital Heart Surgery (RACHS-1) method. ${ }^{11,12}$ All blood transfusions were infused using filters to prevent transmission of donor white blood cells per hospital policy. The decision to send blood, urine, or sputum samples to the laboratory for culture was made by the managing medical team. For the purpose of this analysis, patients were classified as "infected" if any final culture report was positive and the patient received a course of antibiotics for 5 or more days.

\section{Ex Vivo LPS-Induced TNF- $\alpha$ Production}

Whole blood samples were collected in heparinized tubes after anesthesia induction but before incision (presample), at 1 hour after initiation of $\mathrm{CPB}$, or just before discontinuation of bypass if bypass was less than 1 hour (bypass sample), then as close to 8:00 AM as possible on postoperative days 1,3 , and 5. Blood was not collected if arterial or central venous access was removed or if the managing team determined the patient could not tolerate additional blood sampling. Whole blood stimulation was performed within 60 minutes of blood collection.

The whole blood assay has previously been described in detail. ${ }^{13}$ In brief, $50 \mu \mathrm{L}$ of heparinized whole blood was added to tubes of solution ( $500 \mu \mathrm{L}$ ) containing $500 \mathrm{pg} / \mathrm{mL}$ of LPS from S. abortus equii (Sigma-Aldrich, St Louis, Mo) and incubated at $37^{\circ} \mathrm{C}$ for 4 hours. After centrifugation at $10,000 \mathrm{~g}$ for 5 minutes, the supernatants were stored at $-80^{\circ} \mathrm{C}$. Supernatants were shipped to the Immune Surveillance Laboratory at The Research Institute at Nationwide Children's Hospital and assayed for TNF- $\alpha$ using the Immulite automated chemiluminometer (Siemens Medical Solutions Diagnostics, Los Angeles, Calif).

\section{Serum Cytokine Concentrations}

Four mililiters of whole blood was collected in a BD Vacutainer CPT tube (Becton, Dickinson and Company, Franklin Lake, NJ) and the sample was centrifuged at $1800 \mathrm{~g}$ for 30 minutes. The plasma fraction was collected and stored at $-80^{\circ} \mathrm{C}$ until analyzed. Cytokine concentrations (IL-1 $\beta$, IL-6, IL-8, IL-12, TNF- $\alpha$, IL-13, IL-1ra, and IL-10) were determined in triplicate using the Bio-Rad multiplex suspension array system (Bio-Rad Laboratories, Hercules, Calif) per manufacturer's instructions.

\section{Chromatin Immunoprecipitation}

For 6 randomly chosen patients, the buffy coat from the centrifuged CPT tube containing mononuclear cells was transferred to a separate tube containing $10 \mathrm{~mL}$ of $1 \%$ formaldehyde in RPMI medium. Cells were incubated for 10 minutes at $37^{\circ} \mathrm{C}$ followed by centrifugation at $1000 \mathrm{~g}$ for 5 minutes. Cells were washed twice with phosphate-buffered saline and then lysed in $200 \mu \mathrm{L}$ RIPA buffer (Sigma-Aldrich) containing $25 \mathrm{mmol} / \mathrm{L}$ of tris- $\mathrm{HCl}, \mathrm{pH} 7.6,150 \mathrm{mmol} / \mathrm{L}$ of $\mathrm{NaCl}, 1 \% \mathrm{NP}-40,1 \% \mathrm{so}-$ dium deoxycholate, and $0.1 \%$ sodium dodecyl sulfate with $10 \mu \mathrm{L}$ of Halt Protease Inhibitor Cocktail (Pierce, Rockford, Ill) added for each $1 \mathrm{~mL}$ of buffer. Samples were placed on ice for 5 minutes before storage at $-80^{\circ} \mathrm{C}$.

Cell lysates were sonicated to shear the DNA before chromatin immunoprecipitation (ChIP). The Low Cell Number Immunoprecipitation Kit (Diagenode, Liege, Belgium) was used per manufacture's protocol to perform the ChIP assay. In brief, $100 \mu \mathrm{L}$ of sheared DNA in immunoprecipitation buffer supplied by the manufacturer was added to prewashed protein A magnetic beads with anti-H3K4me 3 and incubated at $4{ }^{\circ} \mathrm{C}$ overnight. An aliquot of nonimmunoprecipitated DNA was used as input DNA. After overnight incubation and 3 washes, proteinase $\mathrm{K}$ was added to the samples, which were then incubated for 30 minutes at $55^{\circ} \mathrm{C}$. Samples were boiled for 10 minutes and centrifuged with supernatants being collected for 
TABLE 1. Characteristics of infected and noninfected patients

\begin{tabular}{|c|c|c|c|}
\hline & No infection $(n=76)$ & Infection $(n=16)$ & $P$ value \\
\hline Age $(\mathrm{mo})^{*}$ & $13.5(4-36)$ & $0.87(0.2-5.3)$ & .001 \\
\hline Weight $(\mathrm{kg})^{*}$ & $7.155(5.6-13.9)$ & $3.48(2.9-7.1)$ & .001 \\
\hline Male $(\%)^{*}$ & 56 & 38 & .3 \\
\hline Duration of hospital stay $(\mathrm{d})^{*}$ & $7(5-11)$ & $33(16-48)$ & .001 \\
\hline Duration of hospital stay before surgery $(\mathrm{d})^{*}$ & $0(0-8)$ & $4.5(0-31)$ & $<.001$ \\
\hline Duration of stay in intensive care unit (d)* & $4(3-5.5)$ & $16(5-38)$ & $<.001$ \\
\hline Duration of mechanical ventilation $(\mathrm{d})^{*}$ & $2(1.5-3)$ & $12.5(3-29)$ & $<.001$ \\
\hline Total duration of CPB (min)* & $69(52-105)$ & $151(116-195)$ & $<.001$ \\
\hline Received intraoperative steroids $(\%) \dagger$ & $3(n=2)$ & $12(\mathrm{n}=2)$ & .27 \\
\hline Received modified ultrafiltration $(\%) \dagger$ & $24(\mathrm{n}=18)$ & $25(n=4)$ & .9 \\
\hline Received postoperative blood transfusion $(\%) \dagger$ & $28(\mathrm{n}=21)$ & $31(\mathrm{n}=5)$ & .78 \\
\hline Amount of blood transfused $(\mathrm{mL} / \mathrm{kg})^{*}$ & $10(10-132)$ & $22(10-71)$ & .57 \\
\hline RACHS-1 score* & $3(2-3)$ & $4(3-5.5)$ & $<.01$ \\
\hline Mortality before hospital discharge $(\%)$ & $0(\mathrm{n}=0)$ & $19(n=3)$ & .002 \\
\hline
\end{tabular}

$\overline{C P B}$, Cardiopulmonary bypass; $R A C H S-1$, Risk Adjustment in Congenital Heart Surgery. *Median values with interquartile ranges. $P$ values determined using Mann-Whitney rank sum test. $\dagger P$ values determined using Fisher exact test.

polymerase chain reaction. The following primers were used to determine the association of $\mathrm{H} 3 \mathrm{~K} 4 \mathrm{me} 3$ histone modification with the IL-10 promoter: human IL-10 promoter -531 to -669 : forward, 5'-GGGACAGCTGAA GAGGTGGA; reverse, 3'-CCTCAAAGTTCCCAAGGAGC. Percent of H3K4me3 at IL-10 promoter was calculated as follows: \%(ChIP/Total input $)=2^{\wedge}[\mathrm{Ct}($ input $)-6.6-\mathrm{Ct}($ Chip $)] \times 100 \%$.

\section{Statistical Analysis}

Statistical significance for parametric data was determined using an unpaired $t$ test for experiments comprising 2 groups and a 1-way analysis of variance for experiments comprising 3 or more groups. The Mann-Whitney rank sum test was used to determine statistical significance for nonparametric data. Patients were categorized as immunoparalyzed or immunocompetent and Fisher's exact test was used to determine significance. Whole blood assay test characteristics as well as receiver-operator curves were computed. Statistical tests were conducted using Sigma Stat 3.0 (Aspire Software International, Ashburn, Va), and data were plotted using GraphPad Prism 5.01 (GraphPad Software, Inc, La Jolla, Calif).

\section{RESULTS \\ Patient Characteristics}

Ninety-two patients were enrolled with a median age of 10 months (range, 6 days to 16 years), median weight of $6.8 \mathrm{~kg}$ (range, $1.6-76.2 \mathrm{~kg}$ ), median duration of intensive care unit stay of 4 days (range, 1-98 days), median days on the ventilator of 2 (range, 1-98 days), median duration of hospital stay of 8 days (range, 4-98 days), and an overall mortality of $3 \%$. The median duration of CPB for all patients was 76 minutes (range, 20-354 minutes). Three patients required $\mathrm{CPB}$ more than once and 6 patients required extracorporeal life support before discharge from the intensive care unit. All 22 patients weighing less than $15 \mathrm{~kg}$ had modified ultrafiltration before being weaned from CPB per standardized protocol. Four patients received intraoperative steroids. Twenty-six patients received blood transfusions after CPB. Two patients had delayed sternal closure. The median RACHS-1 score for all patients was 3 (range, 1-6). At least 1 blood sample was collected on every patient with the highest number of whole blood assays obtained on postoperative day 1.

A total of $16(17 \%)$ patients had positive cultures before hospital discharge. Significant differences between patients who had infections compared with those who did not were consistent with other published reports ${ }^{2,4,14-17}$ (Table 1). Patients in whom infections developed were younger, weighed less, had longer intensive care unit and hospital stays, longer durations in the hospital before the operation, longer durations of $\mathrm{CPB}$, and more complex operations. We found no difference in the percentages of patients who received blood transfusions or the median amount of blood transfused between patients in whom infections developed and those in whom they did not (Table 1). Three patients died before hospital discharge and all had positive cultures, for a mortality rate in the infected group of $19 \%$ compared with $0 \%$ mortality for patients with no positive cultures. Details of the positive cultures are listed in Table E1.

\section{Whole Blood Assay on Postoperative Day 1}

Patients were immune phenotyped using an ex vivo whole blood LPS-stimulation assay. Percentages of patients having a whole blood assay performed at each study time point are shown in Figure 1. Whole blood ex vivo TNF- $\alpha$ production was lower for all time points in patients in whom infections developed compared with patients in whom they did not develop (Figure 2) and reached significance for pre-CPB $(P=.045)$ and postoperative day 1 samples $(P=.004)$. We next investigated whether patients who were immunoparalyzed were at a higher risk for postoperative infections. Consistent with previous reports in critically ill pediatric patients, ${ }^{13}$ we defined immunoparalysis as whole blood assay TNF- $\alpha$ production of less than $200 \mathrm{pg} / \mathrm{mL}$. Postoperative day 1 samples were able to discriminate patients for risk of postoperative infections 


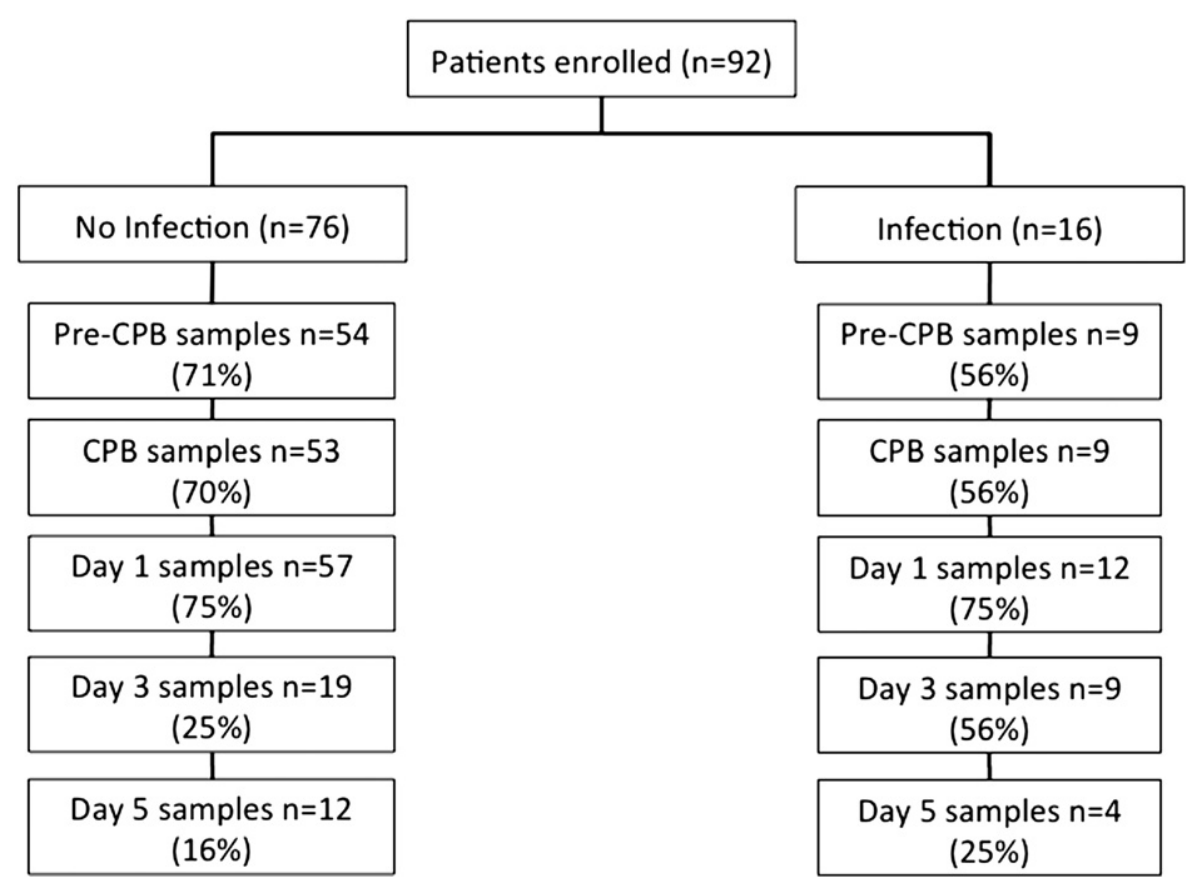

FIGURE 1. Whole blood assays obtained at the study time points for infected and noninfected cohorts. Percentages are the percent of patients in a cohort (infection or no infection) who had a sample obtained at each study time point. $C P B$, Cardiopulmonary bypass.

$(P=.006)$. For the 69 patients in whom we obtained day 1 whole blood assays, $5(42 \%)$ of 12 patients in whom infections developed and $4(7 \%)$ of 54 patients in whom they did not develop were classified as immunoparalyzed. Using a cutoff of $200 \mathrm{pg} / \mathrm{mL}$ for day 1 samples (area under the curve, 0.75; confidence interval (CI), 0.61-0.80), the whole blood assay had a sensitivity of $42 \%$ (CI, 15-72), specificity of $93 \%$ (CI, 83\%-98), positive predictive value of $56 \%(\mathrm{CI}$, $21 \%-86 \%$ ), negative predictive value of $88 \%$ (CI, $77 \%$ $95 \%$ ), and a likelihood ratio of 5.9 for development of a postoperative infection. Comparison of receiver-operator curves using cutoffs ranging from 100 to $500 \mathrm{pg} / \mathrm{mL}$ did not

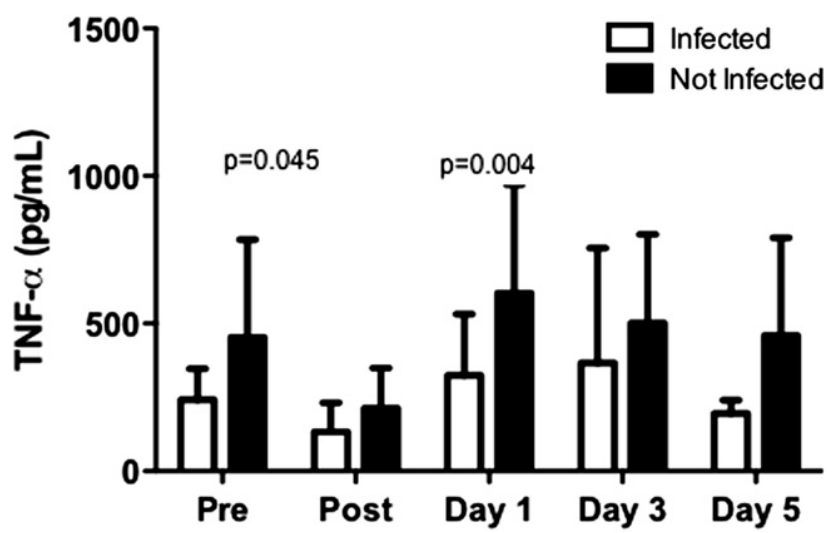

FIGURE 2. Decreased whole blood production of TNF- $\alpha$ after LPS stimulation in ex vivo assay before and on postoperative day 1 in patients in whom patients developed compared with patients in whom infections did not develop. $T N F$ - $\alpha$, tumor necrosis factor-alpha; $L P S$, lipopolysaccharide. improve the test characteristics of the assay. These data suggest that the whole blood assay with a cutoff of $200 \mathrm{pg} / \mathrm{mL}$ is able to reasonably dichotomize patients on postoperative day 1 as immunoparalyzed or immunocompetent and can reliably exclude patients who are at low risk for infections.

\section{IL-10 Levels Were Increased in Immunoparalyzed Patients}

Increased IL-10 levels occur after CPB and have been associated with immunoparalysis. ${ }^{8}$ We similarly observed that immunoparalyzed patients had significantly increased IL-10 production (mean, $14.3 \mathrm{pg} / \mathrm{mL}$ ) as compared with the nonimmunoparalyzed cohort (mean, $6.0 \mathrm{pg} / \mathrm{mL}$; $P=.01$ ) (Figure 3, A). We detected no difference in other selected serum cytokines concentrations (IL-1 $\beta$, IL-6, IL-8, IL-12, TNF- $\alpha$, IL-13, or IL-1ra) on postoperative day 1 between immunoparalyzed and immunocompetent patients (Figure 3, $B$ ). In patients who had both serum samples and whole blood assay on postoperative day 1 ( 9 patients with infection, 47 patients with no infection), the serum IL-10 levels were not above $10 \mathrm{pg} / \mathrm{mL}$ in patients who did not have infections whereas higher serum IL-10 levels correlated with lower TNF- $\alpha$ production in patients who did have infections (Figure E1).

\section{CPB May Affect Histone Modifications in Blood Mononuclear Cells}

Inasmuch as prior work demonstrated that posttranslational histone modification affected IL-10 expression, ${ }^{18}$ 

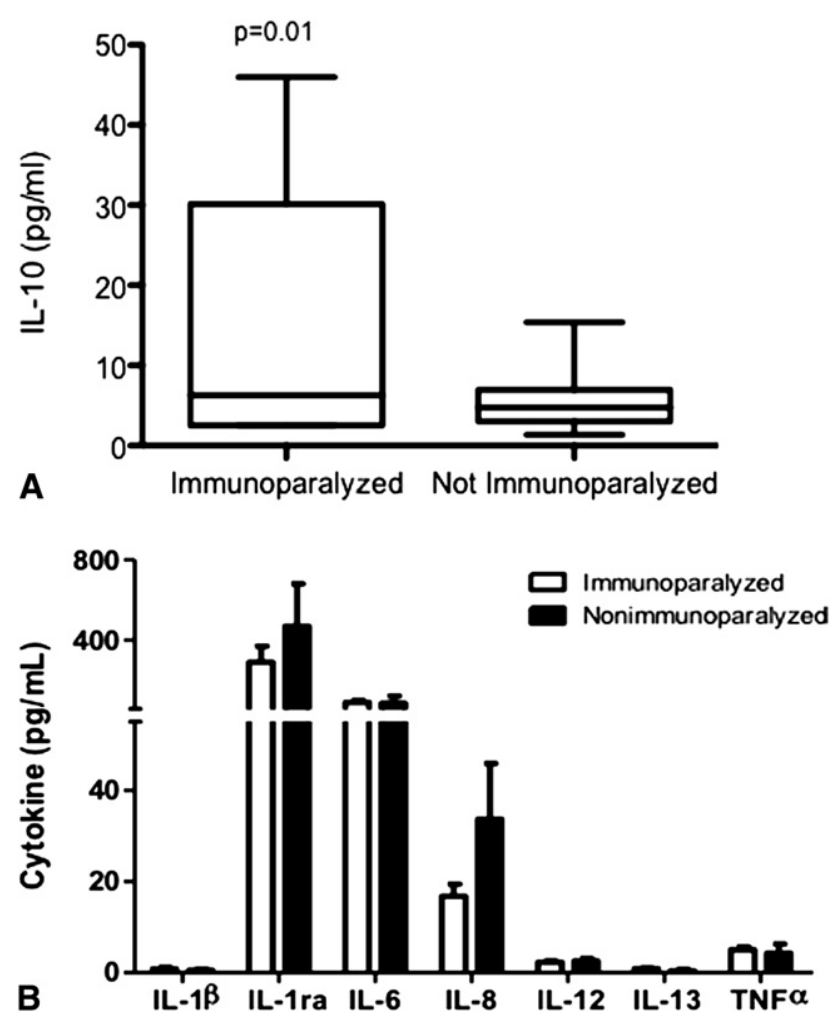

FIGURE 3. Increased serum IL-10 levels in patients identified as immunoparalyzed on postoperative day 1 . Multiplex sampling on serum samples from patients on postoperative day 1 to measure cytokine levels. A, Mean IL-10 serum levels were more than 2-fold higher in patients classified as immunoparalyzed compared with those not immunoparalyzed $(14.3 \mathrm{pg} / \mathrm{mL}$ vs $6.0 \mathrm{pg} / \mathrm{mL} ; P=.01)$. B, No difference detected between immunoparalyzed and nonimmunoparalyzed patients for other serum cytokines measured. $I L$, Interleukin; $T N F-\alpha$, tumor necrosis factor-alpha.

we hypothesized that CPB increased the "gene-on" signature, tri-methylation of lysine 4 on histone 3 (H3K4me3), at the IL-10 promoter, thereby augmenting IL-10 expression.

To investigate the impact of $\mathrm{CPB}$ on the epigenetic signature of patients, we sought to determine whether changes in histone methylation occurred in our patients. We were able to perform ChIP for H3K4me3 on the IL-10 promoter region in only 6 patients who had a median age of 7.5 months (range, 3-72 months), weight $9 \mathrm{~kg}$ (range, 6.1-19.2 kg), RACHS-1 score 2.5 (range, 2-6), and duration of CPB 56 minutes (range, 50-218 minutes). One patient in the cohort had a positive culture and was immunoparalyzed. The results of the ChIP assays indicate a 5-fold increase in the H3K4me3 "gene on" signature at the IL-10 promoter on postoperative day $1(\mathrm{n}=6)$ compared with prebypass signatures (Figure 4). This increase persisted on postoperative day $3(n=4)$. The histone modification, H3K27, was also altered at the IL-12p35 promoter after CPB (data not shown) in the 2 patients of this cohort in whom we were able to obtain adequate samples to detect changes at both

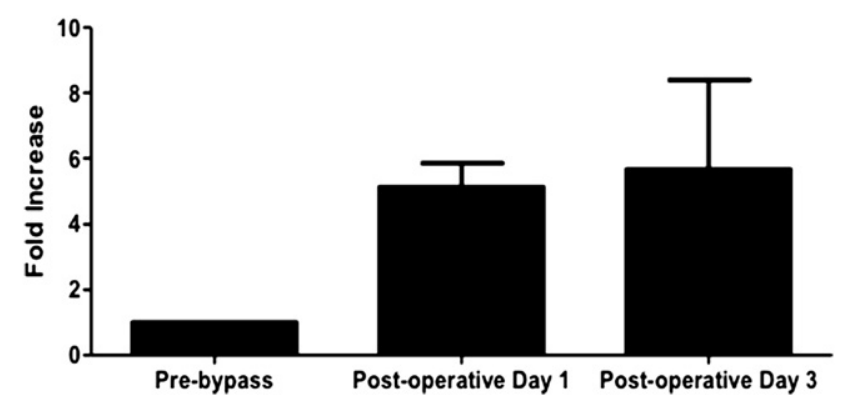

FIGURE 4. Altered posttranslational histone modification, $\mathrm{H} 3 \mathrm{~K} 4 \mathrm{me} 3$, at the IL-10 promoter after CPB. Chromatin immunoprecipitation of $\mathrm{H} 3 \mathrm{~K} 4 \mathrm{me} 3$ followed by polymerase chain reaction IL-10 promoter sites shows a 5-fold increase in the percent of IL-10 promoter associated with the "on" gene histone modification (H3K4me3) on postoperative days 1 and 3 compared with preoperative samples. Values are normalized to each individual patient's prebypass value set at 1 to determine fold change for each postoperative day (postoperative day $1, \mathrm{n}=6$; postoperative day 3 , $\mathrm{n}=4)$. $I L$, Interleukin; $C P B$, cardiopulmonary bypass.

the IL-10 and IL-12 loci. These novel mechanistic data suggest that CPB influences the epigenetic signature at inflammatory gene promoters and illustrate that determining chromatin modifications after CPB is possible in pediatric patients. This novel approach affords the opportunity to further delineate the mechanistic regulation of gene expression characterizing immunoparalysis.

\section{DISCUSSION}

We used a whole blood assay that measured the functional capacity of the immune system to respond to an ex vivo stimulus (LPS), and consistent with the aim of the study, we were able to classify patients as either immunoparalyzed or immunocompetent. Immunocompetent patients (TNF- $\alpha$ concentration $>200 \mathrm{pg} / \mathrm{mL}$ ), on postoperative day 1 , had an $88 \%$ chance of not having a postoperative infection develop. In contrast, immunoparalyzed patients (TNF- $\alpha$ concentration $<200 \mathrm{pg} / \mathrm{mL}$ ) had 2.4-fold higher serum IL-10 levels when compared with immunocompetent patients (Figure 3, A), consistent with the notion that IL-10 expression plays a pivotal role in immunoparalysis and subsequent susceptibility to infections. ${ }^{8}$ Although only examined in a subpopulation of our cohort, we also showed that CPB affects the histone modifications at the IL-10 promoter (Figure 4). Although this sample size was small, these novel data support further investigation into the impact of $\mathrm{CPB}$ on histone methylation. Broader ChIP sequencing should identify additional epigenetically regulated target genes.

The infection rate of $17 \%$ is in the $12 \%$ to $30 \%$ range reported by others for children after CPB. ${ }^{1-3}$ Our cohort with infections was significantly younger and had longer CPB duration, most likely owing to more complex operations as estimated by RACHS-1 scores, compared with patients in whom infections did not develop (see Table 1). Complications associated with postoperative infections 
including increased intensive care unit and hospital stays and increased duration of mechanical ventilation were also observed. We were unable to confirm the association of blood transfusions and increased postoperative infections that other have shown, ${ }^{1,19,20}$ which is most likely a result of the small number of infections noted in our cohort.

A major impact of postoperative infections was a significant mortality rate $(19 \%)$ in the infected cohort, which accounted for all of the deaths in our study population. Thus, our data are consistent with other reports, ${ }^{1-3}$ illustrating a need for early identification of those patients at risk for development of postoperative infections after $\mathrm{CPB}$. The small number of patients with infections in our cohort limits our ability to adequately determine whether immunoparalysis is independent of the other factors associated with postoperative infections. Although identification of patients at low risk for having infections after CPB may allow less exposure to antibiotics, an additional benefit would be to stratify high-risk patients by their immunophenotype in interventional studies targeting immune modulation in patients showing functional immune suppression.

The association of immunoparalysis and infections has been established in critically ill patients. ${ }^{5,7,13,21-23}$ Hall and associates ${ }^{13}$ have shown that the reversal of immunoparalysis in children may prevent the development of secondary infections. Our study adds to a few others that have investigated immunoparalysis in children, including one that investigated the impact of immunoparalysis after CPB. ${ }^{7,8,23,24}$ Allen and colleagues ${ }^{7}$ used flow cytometry to detect cell surface HLA-DR expression on monocytes after CPB and showed a decrease in HLA-DR receptors with the lowest percent of cells expressing HLA-DR receptor occurring at 72 hours after CPB. Inasmuch as monocyte deactivation is a key finding in immunoparalysis and HLA-DR receptor downregulation reflected a "static" measure of monocyte cell surface expression, we sought to investigate a functional characteristic of immunoparalysis: the cytokine production capacity of whole blood in response to LPS. In principle, our findings are consistent with Allen's findings in that immunoparalysis was associated with increased infections, resulting in longer lengths of stays and in our cohort higher mortality. In a subsequent follow-up on their cohort, Allen and colleagues ${ }^{8}$ investigated the role of IL-10 in the development of immunoparalysis and demonstrated that ex vivo production of IL-10 after 24 hours of LPS stimulation of whole blood was augmented.

Results using our whole blood assay on a larger cohort corroborated the data presented by Allen and associates, ${ }^{7}$ inasmuch as we were also able to link immunoparalysis with infections after CPB. Our data argue for the clinical utility of the whole blood assay, inasmuch as it may be more sensitive earlier in the postoperative course as we were able to identify patients on postoperative day 1 as opposed to day 3. Since we detected a decreased ability for ex vivo TNF- $\alpha$ production before CPB (Figure 2), we hypothesize that with a sufficient number of patients sampled before $\mathrm{CPB}$ we may achieve immunophenotyping before CPB. Additionally, our assay has been validated in other critically ill pediatric patients, ${ }^{13}$ allowing us to compare our results to such populations. Our data are also consistent with Allen's findings in that immunoparalysis was associated with increased IL-10 expression (Figure 3, A). Hypothesizing that genetics regulating IL-10 expression may underlie this observation, they also investigated polymorphisms within the IL-10 promoter, but were unable to find a significant difference.

In a mouse model of sepsis, Wen and coworkers ${ }^{25} \mathrm{dem}$ onstrated an alteration in the posttranslational histone modification, H3K4me3, which was associated with altered cytokine production in response to LPS as characterized by decreased proinflammatory cytokine production and, notably, increased IL-10 production. Inasmuch as this pattern of gene expression was similar to that observed in immunoparalysis, we investigated whether CPB had an impact on an epigenetic "gene on" signature at the IL-10 promoter, which may account for the increased IL-10 levels we found. To determine this, we were able to obtain epigenetic signatures from protein-DNA cross-linked samples from selected patients in our cohort. There are several technical limitations in determining such a chromatin-based signature in children, which we were able to overcome for the first time in a limited pediatric cohort. Perhaps the most significant restriction is the volume of blood available for research purposes - and thus the total number of cells available for chromatin isolation. The methodology we used included technical modifications that enabled this isolation from small cell numbers via Low Cell Number Immunoprecipitation Kit (Diagenode, Liege, Belgium). Optimization of antibody immunoprecipitation, IL-10 promoter site determination, and polymerase chain reaction amplification were necessary to achieve the histone methylation changes we identified. To our knowledge, this is the first demonstration of an epigenetically regulated effect obtained from circulating cells in a pediatric cohort. However, inasmuch as the primary goal of this study was to investigate the relationship of immunoparalysis with infections, we were unable to directly associate the impact of changes in histone methylation with immunoparalysis. Our data demonstrate the feasibility of studying the impact of CPB, as well as other inflammatory triggers, on histone modification and the subsequent effect on simultaneously observed gene expression patterns. Another limitation to our study is the small number of patients who had infections, thus resulting in a wide CI for our test parameters. We are currently conducting investigations within the Trial of Euglycemia in Cardiac Surgery (NCT00443599) that is enrolling a cohort of children undergoing $\mathrm{CPB}$ to better delineate the 
relationship between epigenetic processes, immunoparalysis, and the development of infections after surgery.

In summary, our whole blood assay can categorize pediatric patients as immunoparalyzed or immunocompetent on postoperative day 1 , identifying individuals at risk for the development of infections. We have also provided novel data suggesting that CPB may affect cytokine gene expression through an epigenetic mechanism. Further studies are necessary to investigate the relationship between epigenetics and immunoparalysis, as well as the ability to alter the risk for postoperative infections in those patients identified as immunoparalyzed.

We thank Monica Weber and Lauren Conlin for their help with patient enrollment and sample collection, as well as a dedicated nursing staff who assisted in sample collection while providing excellent patient care.

\section{References}

1. Costello JM, Graham DA, Morrow DF, Morrow J, Potter-Bynoe G, Sandora TJ, et al. Risk factors for surgical site infection after cardiac surgery in children. Ann Thorac Surg. 2010;89:1833-41; discussion 1841-2.

2. Grisaru-Soen G, Paret G, Yahav D, Boyko V, Lerner-Geva L. Nosocomial infections in pediatric cardiovascular surgery patients: a 4-year survey. Pediatr Crit Care Med. 2009;10:202-6.

3. Mehta PA, Cunningham CK, Colella CB, Alferis G, Weiner LB. Risk factors for sternal wound and other infections in pediatric cardiac surgery patients. Pediatr Infect Dis J. 2000;19:1000-4.

4. Levy JH, Tanaka KA. Inflammatory response to cardiopulmonary bypass. Ann Thorac Surg. 2003;5:S715-20.

5. Volk HD, Reinke P, Krausch D, Zuckermann H, Asadullah K, Müller JM, et al. Monocyte deactivation-rationale for a new therapeutic strategy in sepsis. Intensive Care Med. 1996;22(Suppl 4):S474-81.

6. Volk HD, Reinke P, Docke WD. Clinical aspects: from systemic inflammation to "immunoparalysis." Chem Immunol. 2000;74:162-77.

7. Allen ML, Peters MJ, Goldman A, Elliott M, James I, Callard R, et al. Early postoperative monocyte deactivation predicts systemic inflammation and prolonged stay in pediatric cardiac intensive care. Crit Care Med. 2002;30:1140-5.

8. Allen ML, Hoschtitzky JA, Peters MJ, Elliott M, Goldman A, James I, et al. Interleukin-10 and its role in clinical immunoparalysis following pediatric cardiac surgery. Crit Care Med. 2006;34:2658-65.

9. Shanley TP, Cvijanovich N, Lin R, Allen GL, Thomas NJ, Doctor A, et al. Genome-level longitudinal expression of signaling pathways and gene networks in pediatric septic shock. Mol Med. 2007;13:495-508.
10. Carson WF 4th, Cavassani KA, Dou Y, Kunkel SL. Epigenetic regulation of immune cell functions during post-septic immunosuppression. Epigenetics. 2011;6: 8-18.

11. Jenkins KJ, Gauvreau K. Center-specific differences in mortality: preliminary analyses using the Risk Adjustment in Congenital Heart Surgery (RACHS-1) method. J Thorac Cardiovasc Surg. 2002;124:97-104.

12. Jenkins KJ, Gauvreau K, Newburger JW, Spray TL, Moller JH, Iezzoni LI. Consensus-based method for risk adjustment for surgery for congenital heart disease. J Thorac Cardiovasc Surg. 2002;123:110-8.

13. Hall MW, Knatz NL, Vetterly C, Tomarello S, Wewers MD, Volk HD, et al. Immunoparalysis and nosocomial infection in children with multiple organ dysfunction syndrome. Intensive Care Med. 2010;37:525-32.

14. Mrowczynski W, Wojtalik M, Zawadzka D, Sharma G, Henschke J, Bartkowski R, et al. Infection risk factors in pediatric cardiac surgery. Asian Cardiovasc Thorac Ann. 2002;10:329-33.

15. Brown KL, Ridout DA, Goldman AP, Hoskote A, Penny DJ. Risk factors for long intensive care unit stay after cardiopulmonary bypass in children. Crit Care Med. 2003;31:28-33.

16. Dagan O, Cox PN, Ford-Jones L, Ponsonby J, Bohn DJ. Nosocomial infection following cardiovascular surgery: comparison of two periods, 1987 vs. 1992. Crit Care Med. 1999;27:104-8.

17. Pollock EM, Ford-Jones EL, Rebeyka I, Mindorff CM, Bohn DJ, Edmonds JF, et al. Early nosocomial infections in pediatric cardiovascular surgery patients. Crit Care Med. 1990;18:378-84.

18. Wen H, Schaller MA, Dou Y, Hogaboam CM, Kunkel SL. Dendritic cells at the interface of innate and acquired immunity: the role for epigenetic changes. J Leukoc Biol. 2007;83:439-46.

19. Costello JM, Graham DA, Morrow DF, Potter-Bynoe G, Sandora TJ, Laussen PC. Risk factors for central line-associated bloodstream infection in a pediatric cardiac intensive care unit. Pediatr Crit Care Med. 2009;10:453-9.

20. Sohn AH, Schwartz JM, Yang KY, Jarvis WR, Guglielmo BJ, Weintrub PS. Risk factors and risk adjustment for surgical site infections in pediatric cardiothoracic surgery patients. Am J Infect Control. 2010;38:706-10.

21. Abe R, Hirasawa H, Oda S, Sadahiro T, Nakamura M, Watanabe E, et al. Up-regulation of interleukin-10 mRNA expression in peripheral leukocytes predicts poor outcome and diminished human leukocyte antigen-DR expression on monocytes in septic patients. J Surg Res. 2008;147:1-8.

22. Monneret G, Lepape A, Voirin N, Bohé J, Venet F, Debard AL, et al. Persisting low monocyte human leukocyte antigen-DR expression predicts mortality in septic shock. Intensive Care Med. 2006;32:1175-83.

23. Hall MW, Gavrilin MA, Knatz NL, Duncan MD, Fernandez SA, Wewers MD. Monocyte mRNA phenotype and adverse outcomes from pediatric multiple organ dysfunction syndrome. Pediatr Res. 2007;62:597-603.

24. Marquardt DJ, Knatz NL, Wetterau LA, Wewers MD, Hall MW. Failure to recover somatotropic axis function is associated with mortality from pediatric sepsis-induced multiple organ dysfunction syndrome. Pediatr Crit Care Med. 2010;11:18-25.

25. Wen H, Dou Y, Hogaboam CM, Kunkel SL. Epigenetic regulation of dendritic cell-derived interleukin-12 facilitates immunosuppression after a severe innate immune response. Blood. 2008;111:1797-804. 


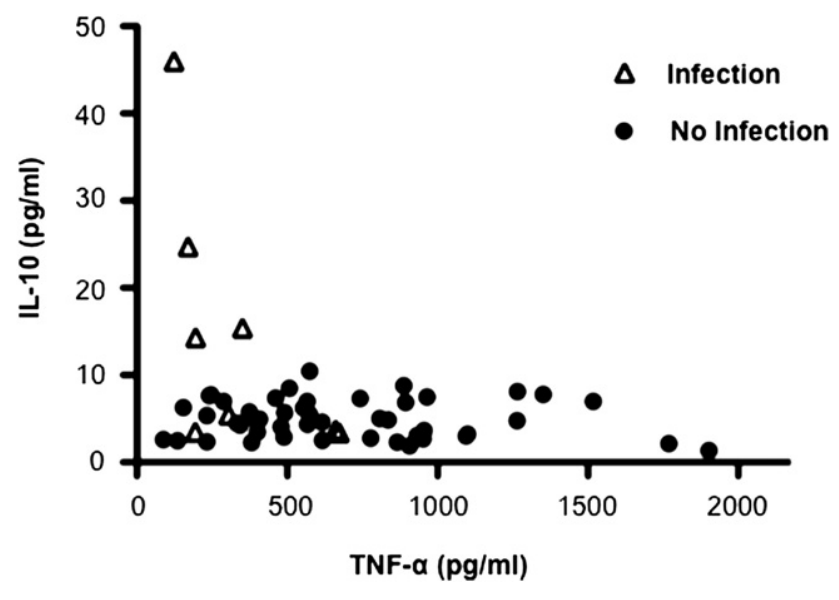

FIGURE E1. Attenuated TNF- $\alpha$ production in whole blood assay is associated with increased serum IL-10 levels. Scatter plots of serum IL-10 levels and TNF- $\alpha$ induction in the whole blood stimulation assay for patients with both samples obtained on day 1 ( $\mathrm{n}=9$ for patients in whom an infection developed, $\mathrm{n}=47$ for patients in whom an infection did not develop). $I L$, Interleukin; $T N F-\alpha$, tumor necrosis factor-alpha.

TABLE E1. Characteristics of positive cultures

\begin{tabular}{llcc}
\hline \multicolumn{1}{c}{ Site } & \multicolumn{1}{c}{ Organism } & $\begin{array}{c}\text { Postoperative day sample } \\
\text { was obtained }\end{array}$ & $\begin{array}{c}\text { Day 1 whole blood } \\
\text { assay }<\mathbf{2 0 0} \text { pg/mL }\end{array}$ \\
\hline Urine & Escherichia coli & 14 & Yes \\
Blood & Staphylococcus aureus & 10 & N/A* \\
Surgical site & Enterobacter cloacae & 3 & No \\
Sputum & Enterobacter cloacae & 8 & No \\
Urine & Enterococcus faecalis & 1 & Yes \\
Sputum & Serratia marcescens & 1 & N/A* \\
Sputum & Enterobacter cloacaea & 5 & N/A* \\
Surgical site & Pseudomonas aeruginosa & 23 & No \\
Sputum & Serratia marcescens & 16 & No \\
Sputum & Staphylococcus aureus & 2 & No \\
Surgical site & Staphylococcus aureus & 15 & Yes \\
Sputum & Enterobacter cloacae & 3 & No \\
Blood & Staphylococcus aureus & 2 & N/A* \\
Blood & Staphylococcus aureus & 2 & Yes \\
Blood & Enterococcus faecalis & 18 & Yes \\
Sputum & Pseudomonas aeruginosa & 3 & No \\
\hline
\end{tabular}

*Whole blood samples were not obtained on day 1 . 